\title{
Pulsed dose rate brachytherapy as the boost in combination with external beam irradiation in base of tongue cancer. Long-term results from a uniform clinical series
}

\author{
Bengt Johansson, MD, PhD'.4, Leif Karlsson, BSc², Johan Reizenstein, MD'.4, Mathias von Beckerath, MD ${ }^{3.4}$, \\ Lennart Hardell, MD, PhD', Jan Persliden MSc, PhD² \\ 'Department of Oncology, 2Department of Medical Physics, ${ }^{3}$ Department of Otolaryngology, ${ }^{4}$ Head and Neck Oncology Center, \\ Örebro University Hospital and Örebro University, Sweden
}

\begin{abstract}
Purpose: To evaluate long time outcome with regard to local tumour control, side effects and quality of life of combined pulsed dose rate (PDR) boost and hyperfractionated accelerated external beam radiotherapy (EBRT) for primary base of tongue (BOT) cancers.

Material and methods: Between 1994 and 2007, the number of 83 patients were treated with primary T1-T4 BOT cancers. Seven patients (8\%) were T1-2N0 (AJCC stage I-II) and 76 (92\%) patients were T1-2N+ or T3-4N0-2 (AJCC stage III-IV). The mean estimated primary tumour volume was $15.4(1-75) \mathrm{cm}^{3}$. EBRT was given with 1.7 Gy bid to 40.8 Gy to primary tumour and bilateral neck lymph nodes in 2.5 weeks. PDR boost of 35 Gy and a neck dissection in clinical node positive case was performed 2-3 weeks later. The patients were followed for a median of 54 (2-168) months.

Results: The 2-, 5- and 10-years rates of actuarial local control were 91\%,89\% and $85 \%$, overall survival $85 \%, 65 \%$ and $44 \%$, disease free survival $86 \%, 80 \%$ and $76 \%$, respectively. The regional control rate was $95 \%$. Six patients $(7 \%)$ developed distant metastases. A dosimetric analysis showed a mean of $100 \%$ isodose volume of $58.2(16.7-134) \mathrm{cm}^{3}$. In a review of late complications 11 cases of minor (13\%) and 5 of major soft tissue necroses (6\%), as well as 6 cases of osteoradionecroses $(7 \%)$ were found. The patients median subjective SOMA/LENT scoring at last follow up was as follow: grade 0 for pain and trismus, grade 1 for dysphagia and taste alteration, and grade 2 for xerostomia. Global visualanalogue-scale (VAS) scoring of quality of life was 8.

Conclusion: Local and regional tumour control rate was excellent in this treatment protocol. The data shows the PDR boost as at least as effective as published continuous low dose rate (CLDR) results.

Key words: brachytherapy, pulsed dose rate, base of tongue cancer, radiotherapy.

\section{Purpose}

The incidence of base of tongue (BOT) cancer in Sweden is low, but has increased over the last 10 years. There were 46 incident cases in 2007 [1]. Smoking and subclinical HPV infection play certain role in the etiology of oropharyngeal cancers $[2,3]$. No randomized studies were conducted in order to compare different treatment strategies. The treatment protocols are based mainly on traditions and have to deal with the local and regional aspect of the disease. In two papers surgery plus postoperative external beam radiotherapy (EBRT) with combined EBRT plus brachytherapy (BT) boost were compared. Local control and survival were similar but they presented better functional performance with EBRT+BT $[4,5]$. The full dose of EBRT alone is also a feasible alternative [6] in the primary treatment. Some authors claimed that BT boost might be a superior option in the most advanced tumours (T3 and T4) [7]. Up till now, the brachytherapy experience has been reported mainly for low dose rate (LDR) manual Ir-wire/ ribbon afterloading [8-11]. Manual afterloading is nowadays more or less discarded due to radiation exposure problems, thus afterloading with a high dose rate (HDR) stepping source for HDR and pulsed dose rate (PDR) fractionation are the modern options. Only a small number of papers reports long time outcome with modern PDR [12] or HDR [13] brachytherapy. The PDR brachytherapy was introduced around 1990 as a substitute for LDR. Small pulses of HDR are given hourly or every second hour,
Address for correspondence: Bengt Johansson, MD, PhD, Department of Oncology, Örebro University Hospital, SE-70185 Örebro, Sweden, Phone: +46 19 6022791, e-mail: bengt.johansson@orebroll.se
Received: 17.02 .11

Accepted: 01.03.11 Published: 31.03 .11 
therefore the overall dose rate and the total dose could be equal to the LDR schedules [14]. We have not found any systematic studies with long time results published on BOT cancer with EBRT and PDR boost.

The aim of this study was to investigate long-term outcome with respect to local tumour control, complications and quality of life in patients with the PDR boost combined with accelerated EBRT. The results were compared with other published data of BOT cancer treatments. Our hypothesis is that PDR brachytherapy proves to be as effective as LDR brachytherapy documented in an earlier experience [15].

\section{Material and methods}

\section{Study cohort}

From the registry of the Head and Neck Oncology Center at Örebro University Hospital we identified a total number of 99 patients with BOT cancer, between December 1994 and September 2007. Of these, 83 patients (83\%) were treated for primary BOT cancer with EBRT+PDR boost + /- elective neck dissection and were included in this retrospective study. The other patients had a treatment for secondary cancers in previously irradiated areas or were unfitted for the combined treatment mentioned above. All patients were selected in a multidisciplinary treatment conference. The staging was based on palpation

Table 1. Clinical data for 83 patients treated for base of tongue cancer

\begin{tabular}{|c|c|c|}
\hline Patient characteristics & $n$ & $\%$ \\
\hline Age & median 60 & $(38-82)$ years \\
\hline Male & 64 & 77 \\
\hline Female & 19 & 23 \\
\hline \multicolumn{3}{|l|}{ TN-stage: } \\
\hline T1NO & 1 & 1 \\
\hline T1N1-2 & 7 & 8 \\
\hline T2NO & 6 & 7 \\
\hline T2N1-3 & 35 & 42 \\
\hline T3NO & 4 & 5 \\
\hline T3N1-2 & 10 & 12 \\
\hline T4NO & 4 & 5 \\
\hline T4N1-3 & 16 & 19 \\
\hline \multicolumn{3}{|l|}{ UICC stage } \\
\hline$|-| \mid$ & 7 & 8 \\
\hline III-IV & 76 & 92 \\
\hline \multicolumn{3}{|l|}{ Histology: } \\
\hline squamous cell carcinoma & 81 & 98 \\
\hline adenoidcystic carcinoma & 2 & 2 \\
\hline \multicolumn{3}{|l|}{ Grade: } \\
\hline low & 9 & 11 \\
\hline medium & 35 & 42 \\
\hline high & 39 & 47 \\
\hline
\end{tabular}

in anesthesia and a computer tomography including the oropharynx, the neck, the thorax and upper abdomen. The proportion of HPV positive patients is unknown. Inclusion criteria were: histology proven primary BOT cancer T1-T4, N0-N3, M0. Patient characteristics are shown in Table 1.

\section{External beam radiotherapy}

At the time we have decided to use accelerated hyperfractionated radiotherapy, since data strongly implied that this type of fractionation was beneficial in the treatment of the head and neck cancers [16-18]. We performed EBRT with 1.7 Gy twice daily to 40.8 Gy in 2.5 weeks covering primary tumour and bilateral neck node areas according to 3D CT based dose plans. Seventy-four patients (89\%) had this treatment schedule. Some referring radiotherapy centers used conventional $2 \mathrm{~Gy} /$ day, fractionation to 46-50 Gy in 5 weeks, thus 9 cases (11\%) were included into this EBRT regimen. Five patients with very advanced locoregional disease had two neoadjuvant cycles of cisplatin (100 mg/sqm day 1) + 5-fluorouracil (1000 mg/sqm day 1-5) and concomitant cisplatin (100 mg/sqm) on day one of EBRT. The start of the treatment was done in median 28 (4-94) days after diagnosis.

\section{Brachytherapy - implant technique}

The time interval between the end of the EBRT and the start of the PDR boost was in median 17 (8-31) days. During that period the patient could recover from acute reaction from EBRT and experienced tumor shrinkage before the brachytherapy and neck surgery phase.

Our aim was to implant the primary volume described from baseline clinical and radiological description. We performed the implantation with a slightly modified technique that was described by Vikram [19] (see Figs. 1A-B). We established this non-looping technique to work very safely in combination with the afterloading machine and a freely movable patient.

The needle entrances were marked on the submental skin and the needles were guided towards the palpating finger on the BOT area. The needles were changed to plastic tubes, which were connected by a suture outside the mouth cavity to the most dorsal catheter going along the BOT surface. The three catheters were then pulled back into the treatment position. We used 6,9 or 12 (median $=9$ ) catheters, depending on the tumour extension. The lateral 3 catheters could also be placed in the lateral wall if there was over-growth into the tonsillar area. The catheters were arranged parallel with 15-20 mm spacing in quadratic configurations according to the rules of Paris system [20]. All patients had a nasogastric feeding tube during the treatment. No patient needed a tracheotomy during the procedure. All patients received antibiotics during the implant procedure, the whole treatment period and seven days after the procedure. The modified Vikram technique is also very easy to handle when the implant is removed. The most anterior buttons are identified and pulled out together with the two connected catheters outside the mouth. When finally each and every catheter are outside the mouth cavity, all catheters can be withdrawn at once with 
A

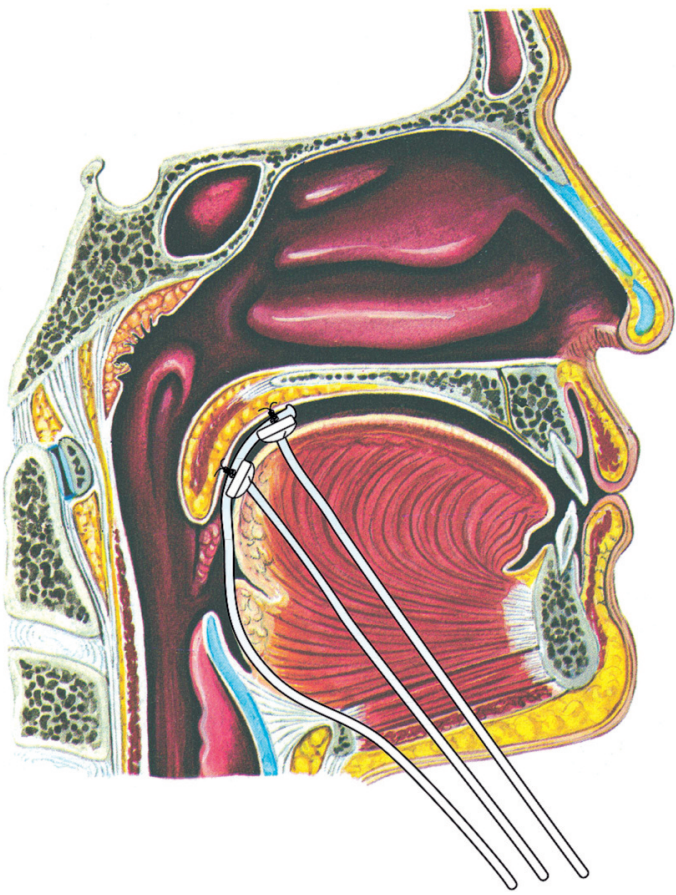

B

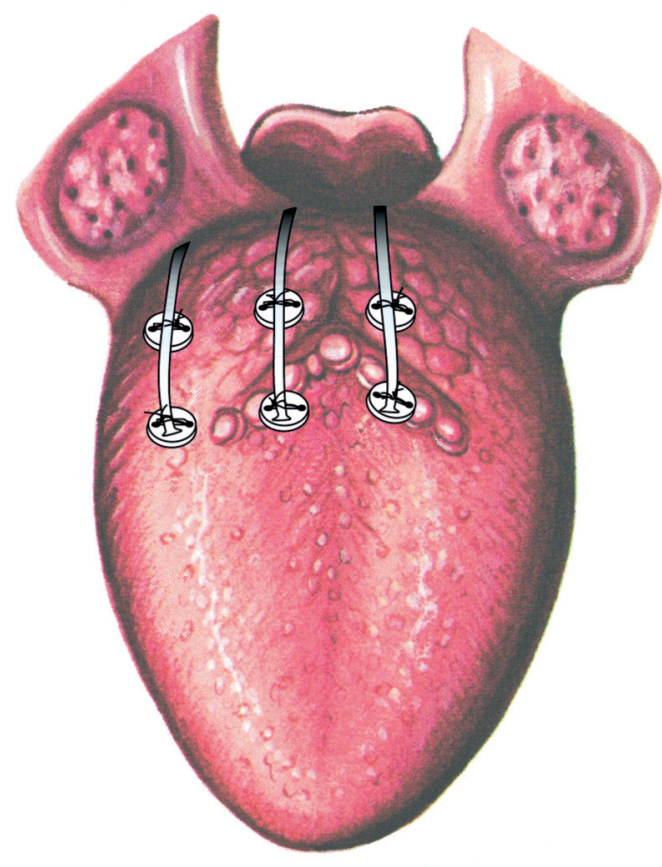

Fig. 1. The implantation with a slightly modified technique that was described by Vikram. A) Lateral view, B) tongue

the patient in drainage position, ensuring any critical bleeding.

\section{Brachytherapy dosimetry}

For the dose calculation, two orthogonal films of the implant were taken and from these, a 3D volume of the implant geometry was reconstructed. The mean central dose (MCD) was the arithmetic mean of the local minimum doses in the dose points placed between the applicators in the central plane, placed according to Paris system. The prescribed dose was the minimum target dose (MTD) to the treated volume and it was defined as $85 \%$ of MCD. The dose distribution was optimized using standard geometrical optimization on volume. The dose calculations were completed with the use of the Nucletron Planning System (NPS, v.10 Nucletron International BV, Veenendaal, the Netherlands). Since March 2006 we gradually introduced 3D CT-based planning (Plato, v.14 Nucletron International BV, Veenendaal, the Netherlands). This procedure was performed in 6 out of 83 patients.

The treatment was applied by pulsed irradiation with HDR Ir-192 source, delivered by PDR microSelectron (Nucletron International BV, Veenendaal, the Netherlands). The prescribed dose was 35 Gy given in 12 pulses per day (with 2 hours interval) over 3.5 days, where the dose per pulse was $0.83 \mathrm{~Gy}$. The group of patients with 46-50 Gy EBRT had a prescribed PDR boost of $30 \mathrm{~Gy}$. We decided to pulse every second hour taking into account both patient convenience and the nursing staff. The outcome of clinical differences and complications would not be expected between hourly and second hourly pulsing from the basic radiobiology publication. All brachytherapy treatments were reviewed and evaluated as recommended by ICRU 58 [21].
A summary of the dosimetry parameters is presented in Table 2.

\section{Radiobiology}

The main rational for this combination of EBRT and brachytherapy was to maintain the overall treatment time short, 35 days in median, in order to complete the whole treatment. The second purpose was to lower the nominal EBRT dose and dose/fraction but still include an effective dosage to tumour targets. The boost dose was increased to improve local tumour control [25]. Table 3 presents biological effective dose (BED) and the equivalent dose in $2 \mathrm{~Gy}$ $\left(\mathrm{EQD}_{2}\right)$ for early and late reacting tissue [26].

Table 2. Dosimetric and volumetric data for treated base of tongue (BOT) cancers

\begin{tabular}{lcc} 
Variable & Mean & Range \\
\hline Estimated BOT tumour volume $\left(\mathrm{cm}^{3}\right)$ & 15 & $1-75$ \\
\hline Treated volume $\left(\mathrm{cm}^{3}\right)$ & 58 & $17-134$ \\
\hline 150\% isodose volume $\left(\mathrm{cm}^{3}\right)$ & 17 & $5-36$ \\
\hline 200\% isodose volume $\left(\mathrm{cm}^{3}\right)$ & 7 & $2-15$ \\
\hline DNR (= V150\%/V100\%) $[22]$ & 0.3 & $0.2-0.4$ \\
\hline Quality index $(\mathrm{QI})[23]$ & 1.7 & $1.3-2.3$ \\
\hline Uniformity index $(\mathrm{UI})[24]$ & 1.5 & $1.3-1.8$ \\
\hline Instantaneoues dose rate $(\mathrm{Gy} / \mathrm{h})$ & 6.0 & $1.0-16.1$ \\
\hline Pulse average dose rate $(\mathrm{Gy} / \mathrm{h})$ & 0.417 & \\
\hline RAKR, reference air kerma rate $\left(\mathrm{mGym}{ }^{2} / \mathrm{h}\right)$ & 2.3 & $0.7-5.5$ \\
\hline TRAK, total reference air kerma $(\mathrm{mGy})$ & 0.34 & $0.15-0.63$
\end{tabular}


Table 3. Biological effective dose (BED) and equivalent dose in 2 Gy $\left(E_{Q} D_{2}\right)$ for: a) EBRT 40.8 Gy $(2 \times 1.7$ Gy/d) + PDR 35 Gy, b) EBRT $25 \times 2$ Gy + PDR 30 Gy, for early and late reacting tissue

\begin{tabular}{llllllll} 
& \multicolumn{2}{c}{ EBRT } & \multicolumn{2}{c}{ PDR } & \multicolumn{2}{c}{ Total } \\
\cline { 2 - 8 } & BED & $\mathrm{EQD}_{2}$ & BED & $\mathrm{EQD}_{2}$ & BED & EQD $_{2}$ \\
\hline a) $\alpha / \beta=3$ & 64.3 & 38.6 & 50.7 & 30.4 & 115.0 & 69.0 \\
\hline b) $\alpha / \beta=3$ & 83.3 & 50.0 & 43.4 & 26.0 & 126.7 & 76.0 \\
\hline a) $\alpha / \beta=10$ & 47.8 & 39.9 & 39.7 & 33.1 & 87.5 & 73.0 \\
\hline b) $\alpha / \beta=10$ & 60.0 & 50.0 & 34.0 & 28.3 & 94.0 & 78.3
\end{tabular}

\section{Neck surgery and pathology}

All patients with clinical lymph node involvement based on fine needle aspiration or radiology findings experienced a neck dissection. Both neck surgery and catheter implant were applied under the same anesthesia. The surgical technique was usually a modified radical neck dissection, however the extent of the surgery was up to the surgeon's decision depending on the extent of lymph node involvement (in some cases bilateral), the age and medical condition of the patient. Adequate sections were stained with haematoxylin-eosin for analysis regarding histopathology. Pathology reports were reviewed to documenter the number of examined and involved lymph nodes.

\section{Follow up}

All patients were examined after 2 to 4 weeks after the procedure to monitor any acute reaction and early complications. The primary tumour response was always judged by at least one examination under anesthesia. After this initial evaluation, the patient was followed by clinical assessment including flexible endoscopy every 3-4 months in the first two years, in the third and fourth year every 6 months and later on annual visits. Minimum 5 years of follow up was mandatory. After this we were able to contact the patient through telephone interviews.

If any adverse event occurred, the patient was examined under general anesthesia and a biopsy or new radiology imaging was performed depending on the clinical presentation in one or multiple sessions. An independent nurse carried out an interview with questions from the symptom module of the LENT SOMA tables [27], a simple visual-analog-scale (VAS) with a patient opinion to estimate quality of life at the last clinical visit or telephone contact of follow up for all living patients 2007-2009.

\section{Statistical methods}

Data from a clinical database was retrieved and analyzed. Local tumour control, disease free survival and overall survival were investigated with the Kaplan-Meier technique. Log-rank test and multivariate Cox regression were performed in order to find factors predicting outcomes such as: T-stage, tumour grade, EBRT fractionation, active smoking, and hemoglobin level at the beginning of brachytherapy. Wilcoxon rank-sum test was performed to evaluate factors related to the risk of radiation necroses. $\chi^{2}$ tests were used to look for correlation between active smoking and necrotic events. All analyses were performed with the Stata SE 10.1 software (Stata/SE 10.1 for Windows, StataCorp, College Station, TX). The study was approved by a Regional Ethical Committee.

\section{Results}

\section{Treatment outcome}

The median clinical follow up time was 46 months (2-147) and contact follow up of 54 months (2-168). No patient was lost in this stage of the study. In total we estimated 9 cases $(11 \%)$ of local failures, out of which seven with T4 tumours. Seven local failures were seen within 15 months. Only one case, a T4 adenoidcystic carcinoma patient, developed very late local recurrence (6.5 years) and was combined with lung metastases. One patient with T4 squamous cell carcinoma developed a very slowly progressing local failure at 28 months and is still alive four years later. Five patients had a salvage surgery and one of them was free of recurrence at autopsy; 1 patient is still alive after one year of salvage surgery. The five-year regional control rate was $95 \%$. In the pathology evaluation of the neck specimen, $41 \%$ was free of tumour cells. Four patients $(5 \%)$ developed regional failures. Two of these patients were earlier treated due to local recurrence. Three patients had a salvage surgery of the neck with a short tumour free survival afterwards. Six patients $(7 \%)$ developed distant metastases. One patient with a single lung metastasis underwent successful resection and is tumour free for 3 years now. Figure 2 shows a graphical KaplanMeier summary of the outcome of the material.

Actuarial local control at 2, 5 and 10 years were 91\%, 89\% and $85 \%$, respectively. The local control rate was dependent on T-stage $(p=0.0005)$, although not for high tumour grade $(p=0.07)$ and hyperfractionated EBRT $(p=0.08)$. Hemoglobin level above or below median $=123 \mathrm{gr} / 1$ did not affect local control rate $(p=0.11)$. Gender $(p=0.10)$ or smoking $(p=0.65)$ did not affect local control rate. In the multivariate Cox regression we found T4-stage $(\mathrm{HR}=11,95 \% \mathrm{CI}=$ $2-57, p=0.01)$ to be the only independent risk factor.

Actuarial disease free survival (DFS) rates at 2, 5 and 10 years were $86 \%, 80 \%$ and $76 \%$, respectively. Actuarial overall survival rates (OS) at 2, 5 and 10 years were $85 \%$, $65 \%$ and $44 \%$, respectively. Active smoking showed a significant worse outcome in the overall survival $(p=0.01)$ but not for DFS $(p=0.09)$. Gender did not affect OS or DFS.

\section{Side effects}

As early side effects (within 3 months) we noticed 4 patients with bleeding at catheter removal. Three patients 
experienced a transient infection in the neck area. Two old patients died early after treatment; 1 male patient died at the age of 79 after 2 month of pneumonia and one 83 years old woman after 3 months of diffuse hypopharyngeal edema and a temporary tracheostomy. In a review of different late events we found 11 minor, transient soft tissue necroses managed by antibiotics. Four patients developed larger, long lasting soft tissue necroses with prolonged conservative antibiotic therapy usually leading to healing. Six patients experienced osteoradionecroses; 4 patients managed by surgery and two by conservative treatment. We looked at all patients with events of necroses and found a statistically significant dependence on the treated volume $(p=0.01)$, but not on the $200 \%$ isodose volume $(p=0.07)$; patients with events of necroses had a larger isodose volume than patients without. We also noticed a tendency, although not statistically significant $(p=0.06)$ that a higher quality index $(\mathrm{QI})$ was related to a risk of necroses. Dose nonuniformity ratio (DNR) $(p=0.26)$, instantaneous dose rate (IDR) $(p=0.93)$ and uniformity index (UI) did not appear to affect the risk of necroses. Active smoking was related to ten major necroses $(p=0.03)$, however not to all necrotic events $(p=0.53)$. In total 8 patients $(10 \%)$ needed a permanent feeding tube or gastrostomia for nutrition. Four patients developed some grade of tongue atrophy many years after the treatment.

\section{SOMA/LENT, quality of life estimate}

We were able to collect data from an interview completed by a nurse with all living patients between March 2007 and March 2009 (summary in Table 4). The median subjective SOMA/LENT patient scoring grade was; 0 for pain (oral/pharyngeal mucosa), 1 (occasional, slight) for taste alteration, 1 (difficulty in eating solid food) for dysphagia, 2 (partial but persistent dryness) for xerostomia (salivary gland), 0 for trismus (mandible). Median weight loss was $12 \mathrm{~kg}$ after treatment and $6 \mathrm{~kg}$ at the last follow up. The median WHO performance was $0(0=$ normal daily activity) and the median visual-analog-scale (VAS) rating of global quality of life was $8(10=$ normal/optimal quality of life) at the last follow-up.

\section{Discussion}

This patient population is representative for clinical practice at a cancer center where the majority of patients experience rather advanced locoregional stage (92\% in UICC stage III-IV). Altered EBRT fractionation was evaluated in meta-analysis [28] and seem to give a significant benefit in locoregional control and survival, compared with conventional fractionation. This was mostly seen with hyperfractionated regimen and in the younger population of patients. The gain with accelerated fractionation was modest, although might be of importance in this setting where there is a gap between EBRT and brachytherapy. Thus, our external beam therapy could be as effective as conventional fractionation in this setting. This is also emphasized in our neck node control rate with $41 \%$ of neck node positive patients free from tumour cells in the pathological evaluation after only $40.8 \mathrm{~Gy}$. In $59 \%$ of the clinical

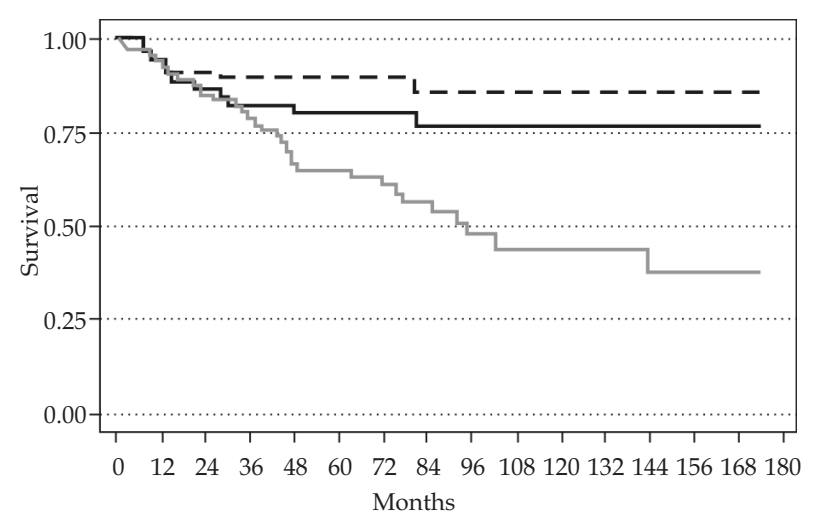

Number at risk

$\begin{array}{lllllllllllllllll}\text { DFS } & 83 & 74 & 57 & 43 & 36 & 31 & 25 & 21 & 13 & 12 & 10 & 9 & 6 & 2 & 1 & 0\end{array}$ Local control

$\begin{array}{llllllllllllllll}83 & 74 & 59 & 46 & 37 & 32 & 25 & 21 & 13 & 12 & 10 & 9 & 6 & 2 & 1 & 0\end{array}$ Overall survival

$\begin{array}{llllllllllllllll}83 & 78 & 61 & 49 & 39 & 33 & 26 & 23 & 14 & 12 & 10 & 9 & 6 & 2 & 1 & 0\end{array}$

—DFS - - Local control _-Overall survival

Fig. 2. Graphical Kaplan-Meier summary of the outcome of the material

neck node positive population there were remaining cancer cells in the neck specimen. Thus, neck dissection cannot be omitted after $40.8 \mathrm{~Gy}$. Our five-year regional control rate $(95 \%)$ was similar to data published by Memorial Sloan-Kettering Cancer Center [29]. Table 5 lists five-year local control and overall survival in different published series and shows that our outcome is similar to the other series with combined EBRT and LDR and HDR brachytherapy. Local control rate, overall and per T-stage, and 5 years survival are similar to the best published data on BOT cancers. In spite of $7 \%$ lower BED and $\mathrm{EQD}_{2}$ with the combination with a shorter overall treatment time (hyperfractionated EBRT and PDR) we noticed a trend for better local control than with longer conventional fractionated EBRT.

Table 4. Patient ratings of subjective SOMA-issues (oral/pharyngeal mucosa, salivary gland, mandible), WHO-performance and global Quality of Life.

Weight changes. Measured at last follow up

\begin{tabular}{lccc} 
Issue & Median & Range & $n$ \\
\hline Pain & grade 0 & $0-3$ & 44 \\
\hline Dysphagia & grade 1 & $0-3$ & 44 \\
\hline Taste alteration & grade 1 & $0-3$ & 44 \\
\hline Xerostomia & grade 2 & $0-4$ & 43 \\
\hline Trismus & grade 0 & $0-2$ & 44 \\
\hline Weight before treatment & $81 \mathrm{~kg}$ & $43-130$ & 43 \\
\hline Weight after treatment & $69 \mathrm{~kg}$ & $38-90$ & 43 \\
\hline Weight last follow up & $75 \mathrm{~kg}$ & $42-106$ & 44 \\
\hline WHO performance & 0 & $0-3$ & 44 \\
\hline Quality of life (VAS) & 8 & $3-10$ & 43
\end{tabular}


Table 5. Clinical outcome data from 10 different institutions on base of tongue cancer treatment

\begin{tabular}{|c|c|c|c|c|c|c|c|c|c|}
\hline \multirow[b]{2}{*}{ Institution } & \multicolumn{9}{|c|}{ Local control (\%) } \\
\hline & $\mathrm{nM}$ & & \%T4 & $\mathrm{T} 1$ & $\mathrm{~T} 2$ & T3 & T4 & All & $5 y S$ \\
\hline Gainesville FL [6] & 217 & $E$ & 19 & 96 & 91 & 81 & 38 & 79 & 50 \\
\hline Curie Inst, Paris, France [30] & 166 & $E$ & 20 & 96 & 57 & 45 & 23 & 44 & 27 \\
\hline IGR, Paris France [10] & 108 & $E+L$ & 0 & 85 & 50 & 69 & nd & 64 & 26 \\
\hline Present series & 83 & $E+P$ & 24 & 100 & 98 & 93 & 65 & 89 & 65 \\
\hline Mayo Clin, Rochester, MN [31] & 79 & $\mathrm{~S}+\mathrm{E}$ & 0 & 84 & 87 & 76 & nd & 82 & 51 \\
\hline Long Beach, CA [11] & 70 & $E+L$ & 17 & 100 & 88 & 75 & 67 & 83 & 35 \\
\hline MSKCC, NY [9] & 68 & $E+L$ & 3 & 87 & 93 & 82 & 100 & 89 & 87 \\
\hline MDA, Houston TX [32] & 54 & $E$ & 2 & 100 & 96 & 67 & nd & 85 & 59 \\
\hline Stanford, CA [8] & 42 & $E+L$ & 24 & 83 & 85 & 89 & 70 & 82 & 66 \\
\hline NIO, Budapest, Hungary [13] & 30 & $\mathrm{E}+\mathrm{H}$ & 53 & - & 100 & 83 & 50 & 68 & 56 \\
\hline
\end{tabular}

$N$ - number of patients, $M$ - method of treatment, $E$ - external beam, L/P/H - LDR/PDR/HDR-brachytherapy boost, $S$ - surgery $\% T 4$ - percentage of T4 in study population, 5 yS - overall five-year survival

Our data like many other reports on radiotherapy of head and neck cancers [33] show a detrimental effect on outcome (OS and DFS) of active smoking during and after the treatment.

The rate of the major soft tissue necroses $(5 \%)$ and osteoradionecroses $(7 \%)$ are similar to other series [7]. Results from Erlangen [34] on PDR brachytherapy of head and neck cancers showed a significant correlation between V85 and the development of soft tissue necroses and similar tendency for the quality index. This is in agreement with our findings concerning treated volume and quality index. With 3D CT-based dose planning we expect that the number of necroses would be reduced. In fact we have not seen any major necroses since we implemented 3D CTbased planning.

There are limited data on functional outcome after BOT treatment. In a large review of the literature it seems clear that surgery and postoperative EBRT yields worse the functional outcome [6]. There are two studies with longterm functional outcome in 30 [4] and 8 patients [5], respectively, evaluated by the performance status scale proposed by List et al. [35]. Eating in public and normalcy of diet was stated around $80 \%$ level $(100 \%$ is normal), which compares well to our SOMA/LENT grade 1 for dysphagia and taste alteration. There is no variable in the SOMA/LENT scale for understandability of speech, which was rated in $90 \%$ level in previous studies $[4,5]$. We noticed only 1 patient with that problem in our material.

Xerostomia was rated $(n=8)$ [5] as 3.3 in the VAS measure $(0=$ no xerostomia) compared to SOMA/LENT grade 2 (partial and persistent) in our series. The median values of the WHO-performance and the global quality of life were very good in our long-term follow up. We noticed a mean of $15 \%(12 \mathrm{~kg})$ drop in weight after the treatment that recovered to a long-term drop of $7 \%(6 \mathrm{~kg})$. The number of patients in long time need of a feeding tube or gastrostomia is higher $(10 \%)$ than in the Stanford experience $(2 \%)$ [8]. We do not have any explanation for this, however it could be related to patient selection factors and possible nutritional ambitions of the treating center.

\section{Conclusions}

This study on PDR boost brachytherapy indicates that PDR fractionation is highly effective and an appropriate substitute for CLDR brachytherapy of head and neck cancers. EBRT or EBRT plus brachytherapy is the treatment of choice for BOT cancer with respect to effectiveness and long-term quality of life.

\section{Acknowledgements}

The Weiderman donation, Svenssons memorial foundation and research committee of Örebro county council supported this work. The authors would like to thank Tony Larsson R.N and Kerstin Kruse R.N for their help in quality of life interviews and to Michael Carlberg MSc for statistical calculations.

\section{References}

1. The national board of health and welfare. Cancer incidence in Sweden 2007. Stockholm: Socialstyrelsen (the national board of health and welfare); 2008 [cited 2009 2009-08-26]. Available from: http:/ / www.socialstyrelsen.se/NR/rdonlyres/64AD2A81 FF9F-459F-B0CF-CA955B7E8513/13410/200812516_rev2.pdf.

2. Schwartz SR, Yueh B, McDougall JK et al. Human papillomavirus infection and survival in oral squamous cell cancer: a population-based study. Otolaryngol Head Neck Surg 2001; 125: $1-9$

3. El-Mofty SK, Patil S. Human papillomavirus (HPV)-related oropharyngeal nonkeratinizing squamous cell carcinoma: characterization of a distinct phenotype. Oral Surg Oral Med Oral Pathol Oral Radiol Endod 2006; 101: 339-345.

4. Harrison LB, Zelefsky MJ, Armstrong JG et al. Performance status after treatment for squamous cell cancer of the base of tongue - a comparison of primary radiation therapy versus primary surgery. Int J Radiat Oncol Biol Phys 1994; 30: 953-957.

5. Van de Pol M, Levendag PC, de Bree RR et al. Radical radiotherapy compared with surgery for advanced squamous cell carcinoma of the base of tongue. Brachytherapy 2004; 3: 78-86.

6. Mendenhall WM, Stringer SP, Amdur RJ et al. Is radiation therapy a preferred alternative to surgery for squamous cell carcinoma of the base of tongue? J Clin Oncol 2000; 18: 35-42. 
7. Foote RL, Parsons JT, Mendenhall WM et al. Is interstitial implantation essential for successful radiotherapeutic treatment of base of tongue carcinoma? Int J Radiat Oncol Biol Phys 1990; 18: 1293-1298.

8. Gibbs IC, Le QT, Shah RD et al. Long-term outcomes after external beam irradiation and brachytherapy boost for baseof-tongue cancers. Int J Radiat Oncol Biol Phys 2003; 57: 489-494.

9. Harrison LB, Lee HJ, Pfister DG et al. Long term results of primary radiotherapy with/without neck dissection for squamous cell cancer of the base of tongue. Head Neck 1998; 20: 668-673.

10. Lusinchi A, Eskandari J, Son Y et al. External irradiation plus curietherapy boost in 108 base of tongue carcinomas. Int J Radiat Oncol Biol Phys 1989; 17: 1191-1197.

11. Puthawala AA, Syed AM, Eads DL et al. Limited external beam and interstitial 192iridium irradiation in the treatment of carcinoma of the base of the tongue: a ten year experience. Int J Radiat Oncol Biol Phys 1988; 14: 839-848.

12. Strnad V, Melzner W, Geiger M et al. Role of interstitial PDR brachytherapy in the treatment of oral and oropharyngeal cancer. A single-institute experience of 236 patients. Strahlenther Onkol 2005; 181: 762-767.

13. Takacsi-Nagy Z, Polgar C, Oberna F et al. Interstitial high-doserate brachytherapy in the treatment of base of tongue carcinoma. Strahlenther Onkol 2004; 180: 768-775.

14. Brenner DJ, Hall EJ. Conditions for the equivalence of continuous to pulsed low dose rate brachytherapy. Int J Radiat Oncol Biol Phys 1991; 20: 181-190.

15. Gerbaulet A, Pötter R, Mazeron J-J et al. (editors). The GEC ESTRO Handbook of Brachytherapy. ESTRO; Brussels 2002.

16. Fowler JF, Lindstrom MJ. Loss of local control with prolongation in radiotherapy. Int J Radiat Oncol Biol Phys 1992; 23: 457-467.

17. Wang CC, Efird JT. Does prolonged treatment course adversely affect local control of carcinoma of the larynx? Int J Radiat Oncol Biol Phys 1994; 29: 657-660.

18. Withers HR, Peters LJ, Taylor JM et al. Local control of carcinoma of the tonsil by radiation therapy: an analysis of patterns of fractionation in nine institutions. Int J Radiat Oncol Biol Phys 1995; 33: 549-562.

19. Vikram B, Hilaris BS. A non-looping afterloading technique for interstitial implants of the base of the tongue. Int J Radiat Oncol Biol Phys 1981; 7: 419-422.

20. Crook J, Mazeron JJ, Marinello G et al. Combined external irradiation and interstitial implantation for $\mathrm{T} 1$ and $\mathrm{T} 2$ epidermoid carcinomas of base of tongue: the Creteil experience (19711981). Int J Radiat Oncol Biol Phys 1988; 15: 105-114.

21. ICRU (editor). Dose and Volume Specification for Reporting Interstitial Therapy. International Commission on Radiation Units and Measurements, Bethesda 1997.

22. Saw CB, Suntharalingam N. Quantitative assessment of interstitial implants. Int J Radiat Oncol Biol Phys 1991; 20: 135-139.

23. Thomadsen BR HP, van der Larse R, Edmundson G, KolkmanDeurloo IK, Visser AG. Treatment planning and optimization. In: Nag S (ed.). High dose rate brachytherapy. A textbook. Futura, New York 1994: 79-145.

24. Anderson LL. A "natural" volume-dose histogram for brachytherapy. Med Phys 1986; 13: 898-903.

25. Wendt CD, Peters LJ, Delclos L et al. Primary radiotherapy in the treatment of stage I and II oral tongue cancers: importance of the proportion of therapy delivered with interstitial therapy. Int J Radiat Oncol Biol Phys 1990; 18: 1287-1292.

26. Dale RG, Huczkowski J, Trott KR. Possible dose rate dependence of recovery kinetics as deduced from a preliminary analysis of the effects of fractionated irradiations at varying dose rates. Br J Radiol 1988; 61: 153-157.

27. LENT SOMA tables. Radiother Oncol 1995; 35: 17-60.
28. Bourhis J, Overgaard J, Audry H et al. Hyperfractionated or accelerated radiotherapy in head and neck cancer: a metaanalysis. Lancet 2006; 368: 843-854.

29. Lee HJ, Zelefsky MJ, Kraus DH et al. Long-term regional control after radiation therapy and neck dissection for base of tongue carcinoma. Int J Radiat Oncol Biol Phys 1997; 38: 995-1000.

30. Jaulerry C, Rodriguez J, Brunin F et al. Results of radiation therapy in carcinoma of the base of the tongue. The Curie Institute experience with about 166 cases. Cancer 1991; 67: 1532-1538.

31. Nisi KW, Foote RL, Bonner JA et al. Adjuvant radiotherapy for squamous cell carcinoma of the tongue base: improved local-regional disease control compared with surgery alone. Int J Radiat Oncol Biol Phys 1998; 41: 371-377.

32. Mak AC, Morrison WH, Garden AS et al. Base-of-tongue carcinoma: treatment results using concomitant boost radiotherapy. Int J Radiat Oncol Biol Phys 1995; 33: 289-296.

33. Fortin A, Wang CS, Vigneault E. Influence of smoking and alcohol drinking behaviors on treatment outcomes of patients with squamous cell carcinomas of the head and neck. Int J Radiat Oncol Biol Phys 2009; 74: 1062-1069.

34. Melzner WJ, Lotter M, Sauer R et al. Quality of interstitial PDRbrachytherapy-implants of head-and-neck-cancers: predictive factors for local control and late toxicity? Radiother Oncol 2007; 82: $167-173$.

35. List MA, Ritter-Sterr C, Lansky SB. A performance status scale for head and neck cancer patients. Cancer 1990; 66: 564-569. 